\title{
Neural Circuitry of Interoception: New Insights into Anxiety and Obsessive-Compulsive Disorders
}

\section{Emily R. Stern, Ph.D}

\author{
Address \\ Department of Psychiatry and Neuroscience, Icahn School of Medicine at \\ Mount Sinai, One Gustave Levy Place, Box 1230, New York, NY 11105, USA \\ Email: emily.stern@mssm.edu
}

Published online: 21 June 2014

(C) Springer International Publishing AG 2014

Keywords OCD - Psychiatric disorders - Interoceptive awareness - Emotional and cognitive processes - Abnormal interoception

\begin{abstract}
Over the past century, much research has investigated how the brain processes signals from the body (interoception) and how this processing may be disturbed in patients with psychiatric disorders. In this paper, I discuss the literature examining the relationship between interoceptive awareness and emotional and cognitive processes, and review the evidence suggesting that anxiety and obsessive-compulsive disorder (OCD) are characterized by abnormal interoception. A network of cortical and subcortical brain regions centered on the insula has repeatedly been implicated in interoception and is abnormal in anxiety and OCD. The investigation of interoception provides a framework for understanding behavioral and neural mechanisms of anxiety and $O C D$, although additional research is needed to directly link insula functioning to aberrant interoception in these disorders. Future work targeting interoception may be useful for the development of novel treatments.
\end{abstract}

\section{Introduction}

Anxiety disorders and obsessive-compulsive disorder (OCD) are common, with a total lifetime prevalence rate of over $28 \%$, and are strongly associated with poor quality of life [1]. Given that current first-line treatments including cognitive behavioral therapy and psychotropic medication are only partially effective [2], further investigation into core behavioral and neural mechanisms will be critical for identifying targets for novel treatments. In the present review, I discuss evidence supporting the theory that abnormal 
awareness of, or sensitivity to, sensations from the body (i.e., interoception) is a core feature of anxiety and OCD. Previously discussed largely in the context of anxiety disorders [3-6], I propose that this framework can also be applied to understanding the phenomenology and neurobiology of OCD and related disorders. In the first half of the review, I describe historical work examining how body signals influence behavior, highlighting the relationship between interoceptive awareness and emotional and cognitive functioning. I then outline research pointing to abnormal interoception among patients with anxiety and OCD. In the second half of the review, I describe the neural circuits responsible for processing information from the body, and link this to what is known about brain functioning in anxiety and OCD. I conclude with some suggestions for future ways to use interoception as a target for treatment.

\section{The relationship between the body and emotion}

The link between body signals and emotional and cognitive processes has been a topic of much interest in psychology and psychiatry. In the late 1800s, the influential scientists William James and Carl Lange independently proposed that emotional stimuli evoke physiologic responses in the viscera and soma, and that it is the perception of these body-state changes that results in the experience of emotion, a hypothesis that has come to be known as the James-Lange Theory of Emotion [7, 8•]. This view has been hotly debated, with some experimental work supporting a causal link between body signals and emotion and others refuting it [7]. Critics of the James-Lange theory offer many plausible arguments against a strict interpretation [7], with one of the most commonly cited criticisms being that physiologic signals are too general to be associated with discrete emotions [9-11]. However, an update to this theory came in the 1960s, when Schacter and Singer [11] proposed that "emotional state may be considered a function of a state of physiologic arousal and of a cognition appropriate to this state of arousal". In their nowfamous experiment, the researchers injected subjects with epinephrine before placing them in a room with a study confederate who acted in an angry or euphoric manner. Some subjects were told about the typical physiologic response to epinephrine (e.g., increased heart rate, sweating, trembling), while others were not told anything about what to expect (the "ignorant" group). Results indicated that subjects who had received epinephrine but who were "ignorant" to its effects were more likely to display emotions consistent with those shown by the confederate as compared with subjects who were informed about the effects. These data suggest that physiologic responses do influence emotional experience, but in a way that depends on contextual and cognitive factors, a theory that may have been closer to James' original concept than is typically appreciated [7]. More contemporary views, such as the somatic marker hypothesis, also emphasize the influence of physiologic signals on cognition and emotion [12, 13].

In addition to the effects of the mere presence of physiologic signals, the propensity or sensitivity to detect such signals - sometimes referred to as interoceptive awareness (IA) - also appears to be important for emotion and cognition $[8 \bullet 14,15]$. There is considerable individual variability in IA, often indexed by accuracy in detecting one's own heartbeat, and greater IA has been consistently associated with increased emotional arousal [14, 16-18]. 
IA is also related to the cognitive processing of emotional stimuli, in that greater IA has been linked to improved decision making under risk [19], greater regulation of emotion [20], improved acquisition of fear conditioning [21], better implicit memory for emotional words [22], better recognition memory for emotional pictures [23], and increased cognitive interference from negative words [24]. In addition, IA has been shown to moderate the relationship between behavior and brain functioning [25] and behavior and physiology [26, 27].

\section{Physical symptoms in anxiety and obsessive compulsive disorder (ocd)}

Given the relationship between body signals and emotion, it is perhaps not surprising that several psychiatric disorders are associated with altered physical sensation. Diagnostic and Statistical Manual for Mental Disorders [28] criteria for many anxiety disorders specify the presence of physical symptoms, particularly for panic disorder (PD), which can involve increased heart rate, sweating, feelings of choking or difficulty breathing, abdominal distress or chest pain, paresthesia, or trembling. In addition, criteria for generalized anxiety disorder (GAD) include restlessness and muscle tension, and a diagnosis of post-traumatic stress disorder (PTSD) requires persistent symptoms of increased physiologic arousal. In line with the inclusion of physical symptoms in diagnostic criteria, patients with anxiety consistently report sweating, increased heart rate, and difficulty breathing at a greater frequency than healthy controls [29-32].

Although not part of the diagnostic criteria, patients with OCD report physical symptoms similar to those described by patients with anxiety [33]. In addition, up to $70 \%$ of patients with OCD experience "sensory phenomena", which are aversive or uncomfortable internal sensations that drive repetitive behaviors $[34 \bullet \bullet, 35,36]$. Sensory phenomena manifest as tactile or visceral sensations of contamination, including sensations of disgust, which precipitate washing/cleaning compulsions. In addition, sensory phenomena may involve uncomfortable sensations in the skin or muscles prior to touching compulsions. Repeating and arranging compulsions are also frequently performed to achieve a specific, or "just right", sensation, which can be physical or mental $[34 \bullet \bullet, 37,38]$. Sensory phenomena are also highly prevalent in Tourette's disorder (TD), where they manifest primarily as muscle tension, numbness, tingling, and/or itching in a specific body part prior to tics, known as a premonitory urge or "sensory tic" [39-41]. In addition, certain impulse control disorders such as trichotillomania and skin picking (excoriation disorder) are characterized by premonitory urges [42].

\section{Interoception in anxiety and ocd}

Despite the greater frequency of physical symptoms reported by patients with anxiety, objective measures of physiologic arousal do not consistently match subjective experiences. Some studies show increased physiologic arousal in patients with anxiety [43], whereas others find no differences from controls 
$[29,32]$. This raises the possibility that anxiety is associated with a heightened sensitivity or bias towards interoceptive information in the absence of major physiologic differences, similar to models proposed for "medically unexplained symptoms" [44]. Indeed, conceptualizations of PD often focus on patients' sensitivity to and interpretation of body signals (see [3] for a review), and certain theories of anxiety propose that patients exhibit an exaggeration of aversive interoceptive signals and have difficulty distinguishing between signals that are associated with negative consequences and those that are not $[5,6]$.

Supporting these theoretical constructs, higher anxiety among healthy individuals has been related to greater IA as assessed by heartbeat detection accuracy $[45,46]$. Patients with PD and GAD also show greater heartbeat detection accuracy than healthy controls (see [4] for a review). In a re-analysis of data from 709 subjects including 275 PD patients, Van der Does [47] found that patients with PD were more accurate at detecting their heartbeat than were healthy controls, patients with heart palpitations, those with infrequent panic attacks, and depressed patients, but were no different from patients with social phobia, specific phobia, and GAD. Subsequent work has confirmed that socially anxious individuals are better at detecting their own heartbeat [48]. Although no studies have yet investigated IA in patients with OCD or TD, it is possible that an increased awareness of or attention to body sensations contributes to the sensory phenomena experienced in these disorders $[49,50]$.

\section{A note on interoceptive accuracy vs. subjective body experiences}

It should be noted that measures of IA that rely on accuracy (such as in detecting one's own heartbeat) may not capture the entire picture of the interoceptive experience, in that individuals may be more sensitive to body sensations without necessarily being more accurate it judging them. Describing this issue, Garfinkel and Critchley [51] distinguish between objective measures of IA that rely on accuracy (what they term "interoceptive sensitivity") and subjective measures of IA that rely on self-report (what they term "interoceptive sensibility"). They emphasize the importance of assessing both objective and subjective measures of interoceptive awareness, given that they are not typically correlated $[44,51]$.

\section{Neural correlates of interoception}

Much research has identified the neural pathways involved in detecting information from the body. Although the term interoception has sometimes been used to refer to the detection of sensations from visceral organs only, a broader conceptualization has been proposed by Craig $[52,53]$ to include sensation from the entire body, including skin, muscles, joints, and teeth in addition to viscera. The majority of signals coming from visceral organs reach the brain via cranial nerves IX and X (glossopharyngeal and vagus nerves) and the dorsal horn of the spinal cord $[54,55 \bullet, 56,57]$, whereas somatic 
information is relayed through the dorsal horn route [52, 53, 57]. Afferents project to the nucleus of the solitary tract in the medulla $[55 \bullet, 56,58]$ and area postrema [59], with parallel and sequential pathways continuing to the parabrachial nucleus, periaqueductal gray, thalamus, and hypothalamus $[52-54,55 \bullet]$. Largely by way of the thalamus, these signals are transmitted to cortical and limbic regions including the insula, somatosensory cortex, cingulate cortex, orbitofrontal cortex, and amygdala [52, 54, 55•]. A broad conceptualization of interoception that includes sensations from the entire body is supported by research showing that visceral and somatic afferents converge at several levels on their way to the brain, including in the dorsal horn of the spinal cord, the nucleus of the solitary tract, and the thalamus $[57,58,60,61]$. Indeed, the co-localization of visceral and somatic terminals on neurons of the dorsal horn has been implicated in referred pain [60,61].

Among the cortical areas involved in interoception, the insula has emerged as a key region. The insula, meaning "island" in Latin, is a triangular area of cortex embedded under the intersection of the frontal, temporal, and parietal lobes. Electrical stimulation of the insula elicits somatic and visceral sensations $[62,63]$, and insular lesions have been associated with abnormalities of somatosensation and body awareness [64]. The insula extends 50-60 mm antero-posteriorly, with cytoarchitectonic features distinguishing anterior from mid-posterior regions, bifurcated roughly at the central sulcus (for a review see [65]). While neuroimaging studies have found activation of both anterior [45, 66-68] and mid-posterior insula [68-70] during tasks of interoception when subjects are instructed to attend to their body sensations, other research suggests a dissociation of function between these sections [71-73]. Mid-posterior insula, being a major recipient of ascending sensory signals [52, 53], may hold the initial cortical representation of the body (as referred to as "primary interoceptive cortex") that is then re-represented with greater complexity in anterior insula [52, 74]. Anterior insula is activated to a variety of psychological tasks [71, 73, 75 ], and is hypothesized to integrate primary body representations with emotional, cognitive, and motor systems to generate subjective "feeling states" [45, 52, 55•, 69, 74, 76]. Anterior insula contains thin, elongated, spindle-shaped cells called "von Economo neurons" (so-named for one of the early discoverers [77]), which are capable of fast communication over long distances $[65,78]$, supporting the hypothesis that this region is important for neural integration. The dissociation of function between anterior and mid-posterior insula is further supported by functional and structural connectivity studies revealing differing patterns of connectivity between the regions $[79,80]$.

\section{The insula and other body-related processes}

Disgust can be thought of as a feeling state that involves both an emotion and a stereotypical body response that has evolved as a defense to prevent ingestion of toxins or pathogens [81]. The study of disgust is particularly relevant for OCD, as many patients with contamination symptoms identify disgust as a major trigger of their cleaning compulsions [82, 83]. In addition, small animal and blood-injection-injury phobias are prominently charac- 
terized by disgust [84]. Neuroimaging studies of healthy individuals reveal activation of both anterior and mid-posterior insula in response to disgustinducing images [85-87] and facial expressions of disgust [88-90]. In one case study, a patient with a lesion encompassing both anterior and posterior insula was impaired in recognizing facial expressions of disgust but exhibited normal recognition for several other emotions including happiness, fear, and sadness [91]. Although there is continued debate as to whether the insula is involved in processing disgust per se or is generally important for emotion [87], one possibility is that insula activations to various emotional probes are related to physiologic responses.

Another set of body-related processes that are sub-served by the insula are so-called "urges for action", defined as physical sensations that drive an individual to perform a behavior [92•]. Urges can be unconscious or conscious, and are typically automatic and habitual [92•]. Everyday examples include the urge to blink, the urge to scratch an itch, and the urge to cough. In patients with $\mathrm{OCD}$ or $\mathrm{TD}$, sensory urges commonly precede compulsive behavior or tics $[34 \bullet \bullet, 35,36,92 \bullet]$. A small body of neuroimaging work has investigated neural activation during the build-up of urges, with findings consistently pointing to the insula as well as other regions linked to interoceptive and motor functions. In a recent review, Jackson and colleagues (2011) noted a common region of posterior insula during the urge to swallow, urge to yawn, and urge to urinate, and, in TD patients (originally reported by [93]), during the urge to tic. It has been proposed that the physical experience of an urge is processed in secondary somatosensory cortex and mid-posterior insula, while the conscious awareness of the urge involves anterior insula (Jackson 2011). Although, to my knowledge, no studies have yet examined neural differences between conscious and unconscious urges, this hypothesis would be consistent with the proposed anterior-posterior segregation of insular function already described $[45,52,69,74]$.

\section{Insula functioning in anxiety and OCD}

Several studies have found that patients with anxiety disorders exhibit abnormal functioning of the insula, the details of which have been described by other authors $[5,6,94]$. A meta-analysis [95] comparing patients with PTSD, social anxiety disorder, and specific phobia with healthy controls identified insula hyperactivity in all three disorders across a variety of tasks, most of which presented stimuli designed to provoke symptoms (e.g., reading trauma scripts, looking at images of phobic stimuli). In healthy individuals, chemically induced panic after injection of cholecystokinin- 4 elicited a robust bilateral insula response that was correlated with self-rated panic severity [96]. A study in PD also found that anterior insula was activated more in patients who experienced a lactate-induced panic attack than in patients who did not experience a panic attack. Intriguingly, Cameron [97] found reduced binding of receptors for the inhibitory neurotransmitter $\gamma$ aminobutyric acid $\mathrm{A}_{\mathrm{A}}\left(\mathrm{GABA}_{\mathrm{A}}\right)$ in bilateral mid-posterior insula in PD patients, suggesting that impaired inhibitory functioning in the insula may contribute to the observed hyperactivity. 
Considerable research has also identified increased activation and connectivity of the insula in patients with $\mathrm{OCD}$, who show greater resting-state metabolism of mid-posterior insula than controls [98] and increased restingstate connectivity within anterior insula [99], as well as between anterior and posterior insula [100]. During symptom provocation, OCD patients show activation of anterior insula [101, 102]; similarly, studies focusing on disgust processing have identified hyperactivity in anterior [103, 104] and midposterior [104, 105] insula when patients view disgusting images. Further, several paradigms exploring brain functioning in OCD when patients perform cognitive tasks not directly related to symptoms have identified hyperactivity in the insula, including tasks of error detection [106], conflict detection [107], decision making [108], planning [109], response inhibition [110] working memory [111], fearful face processing [112], loss anticipation $[113,114]$, reward [114], and negative performance feedback [115]. Although hyperactivations in these studies are more frequently reported in anterior insula [106-109, 112-114] than in mid-posterior insula [110, 111, $114,115]$, there are no readily apparent psychological or cohort factors that explain the anterior-posterior differences among studies. It is possible that the various paradigms that have been used, not having been designed to specifically measure interoception, were not sensitive to differences in primary interoceptive processes (supposedly processed in mid-posterior insula) as opposed to higher-order integrative functions of the insula (purportedly subserved by anterior insula). Given that anterior insula is associated not only with interoception but also with emotion and cognition [71, 73, 75], the use of task paradigms that directly tap into interoceptive processes in anxiety and OCD would aid in the interpretation of neuroimaging findings.

It should be noted that some studies have also reported hypoactivations of the insula in OCD compared with controls, with the majority of this work focusing on brain activity during task switching [116-118]. These findings highlight the importance of considering the specific task used to investigate brain functioning when interpreting results. To my knowledge, no studies have yet examined neural activity in patients with anxiety disorders or OCD when directly engaging interoceptive processes. However, one study in healthy individuals found a correlation between anterior insula activity and trait anxiety (measured with the Hamilton Anxiety Rating Scale) when subjects attended to their own heartbeat [45]. In this study, anxiety was also correlated with IA as assessed by heartbeat detection accuracy. These results are important in that they provide a convincing link between increased insula activity and altered IA in relation to psychopathology.

\section{Conclusions and future steps}

Over the past 100+ years, the study of how the brain detects signals from the body, and how this interacts with emotion and cognition, has yielded considerable insight into psychiatric disorders including anxiety and OCD. Research has identified a neural circuit cortically centered on the insula that is involved in interoception and similar body-related processes such as disgust perception and urges for action. Patients with anxiety disorders and OCD show abnormal hyperactivity of the insula in a variety of tasks, which could 
be related to altered interoception, although studies directly probing interoception in patients will be necessary to test this hypothesis.

Arguably, one of the major goals of psychiatric research is to identify behavioral and brain targets for treatment, and there are several ways that interoception can serve as such a target. Exposure to interoceptive cues is frequently used to reduce sensitivity to body sensations in panic disorder $[119,120]$, an approach that could be useful for other anxiety disorders as well as OCD. In addition, neural mechanisms associated with altered interoception can be targeted by brain stimulation techniques such as deep brain stimulation and transcranial magnetic stimulation, which have already been employed to modulate neural functioning in psychiatric and neurologic disorders [121], including OCD [122-125]. Neurobiologic targets can also be modulated using real-time functional magnetic resonance imaging (fMRI), which is a developing technology that trains individuals to modify activity in specific brain regions using neurofeedback of the fMRI, blood-oxygen level-dependent signal [126]. Several studies have successfully trained subjects to increase activity in anterior insula using real-time fMRI neurofeedback [127-129], although decreasing activity in this region may prove more difficult [129]. Finally, novel drug therapies may be developed to modulate specific circuits or neurotransmitter systems suspected to be involved in interoception. For example, the GABA system may be a promising target, as GABA concentration is linked to interoceptive awareness [68] and GABA receptor binding is reduced in bilateral insula in PD patients [97]. In conclusion, the investigation of interoception in anxiety and OCD has provided new insights into behavioral and neural mechanisms, which could be used as future targets for treatment.

\section{Compliance with Ethics Guidelines}

\section{Conflict of Interest}

Emily R. Stern discloses a grant paid by the National Institute of Health.

Human and Animal Rights and Informed Consent

This article does not contain any studies with human or animal subjects performed by any of the authors.

\section{References}

Papers of particular interest, published recently, have been highlighted as:

- Of importance

$\bullet \quad$ Of major importance

1. Olatunji BO, Cisler JM, Tolin DF. Quality of life in the anxiety disorders: a meta-analytic review. Clin Psychol Rev. 2007;27(5):572-81.

2. Ballenger JC. Current treatments of the anxiety disorders in adults. Biol Psychiatry. 1999;46(11):157994. 
3. Bouton ME, Mineka S, Barlow DH. A modern learning theory perspective on the etiology of panic disorder. Psychol Rev. 2001;108(1):4-32.

4. Domschke K, Stevens S, Pfleiderer B, et al. Interoceptive sensitivity in anxiety and anxiety disorders: an overview and integration of neurobiological findings. Clin Psychol Rev. 2010;30(1):1-11.

5. Paulus MP, Stein MB. An insular view of anxiety. Biol Psychiatry. 2006;60(4):383-7.

6. Paulus MP, Stein MB. Interoception in anxiety and depression. Brain Struct Funct. 2010;214(5-6):45163.

7. Friedman BH. Feelings and the body: The Jamesian perspective on autonomic specificity of emotion. Biol Psychol. 2010;84(3):383-93.

8. Herbert BM, Pollatos O. The Body in the Mind: On the Relationship Between Interoception and Embodiment. Top Cogn Sci. 2012;4(4):692-704.

This provides an overview of models of interoception and "embodied" cognition, and highlights the relevance of interoception for certain psychopathological states.

9. Bard P. Emotion I. The neuro-humoral basis of emotional reactions. In: Murchsion $\mathrm{C}$, editor. The Foundations of Experimental Psychology. Worcester: Clark University Press; 1929. p. 449-87.

10. Cannon WB. The James-Lange theory of emotion: a critical examination and alternative theory. Am J Psychol. 1927;39:106-24.

11. Schachter S, Singer JE. Cognitive, Social, and Physiological Determinants of Emotional State. Psychol Rev. 1962;69(5):379-99.

12. Damasio AR. The somatic marker hypothesis and the possible functions of the prefrontal cortex. Philos Trans R Soc B. 1996;351(1346):1413-20.

13. Bechara A, Tranel D, Damasio H, et al. Failure to respond autonomically to anticipated future outcomes following damage to prefrontal cortex. Cereb Cortex. 1996;6(2):215-25.

14. Barrett LF, Quigley KS, Bliss-Moreau E, et al. Interoceptive sensitivity and self-reports of emotional experience. J Pers Soc Psychol. 2004;87(5):684-97.

15. Katkin ES. Blood, Sweat, and Tears - Individual-Differences in Autonomic Self-Perception - PresidentialAddress, 1984. Psychophysiology. 1985;22(2):12537.

16. Herbert BM, Pollatos $\mathrm{O}$, Schandry R. Interoceptive sensitivity and emotion processing: An EEG study. Int J Psychophysiol. 2007;65(3):214-27.

17. Pollatos $\mathrm{O}$, Kirsch W, Schandry R. On the relationship between interoceptive awareness, emotional experience, and brain processes. Cogn Brain Res. 2005;25(3):948-62.

18. Wiens S, Mezzacappa ES, Katkin ES. Heartbeat detection and the experience of emotions. Cogn Emot. 2000;14(3):417-27.
19. Werner NS, Jung K, Duschek S, et al. Enhanced cardiac perception is associated with benefits in decisionmaking. Psychophysiology. 2009;46(6):1123-9.

20. Fustos J, Gramann K, Herbert BM, et al. On the embodiment of emotion regulation: interoceptive awareness facilitates reappraisal. Soc Cogn Affect Neurosci. 2013;8(8):911-7.

21. Katkin ES, Wiens S, Ohman A. Nonconscious fear conditioning, visceral perception, and the development of gut feelings. Psychol Sci. 2001;12(5):366-70.

22. Werner NS, Peres I, Duschek S, et al. Implicit memory for emotional words is modulated by cardiac perception. Biol Psychol. 2010;85(3):370-6.

23. Pollatos O, Schandry R. Emotional processing and emotional memory are modulated by interoceptive awareness. Cogn Emot. 2008;22(2):272-87.

24. Werner NS, Mannhart T, Reyes Del Paso GA, Duschek S. Attention inference for emotional stimuli in cardiac interoceptive awareness. Psychophysiology. 2014;51:573-8.

25. Werner NS, Schweitzer N, Meindl T, et al. Interoceptive awareness moderates neural activity during decision-making. Biol Psychol. 2013;94(3):498-506.

26. Dunn BD, Evans D, Makarova D, et al. Gut feelings and the reaction to perceived inequity: The interplay between bodily responses, regulation, and perception shapes the rejection of unfair offers on the ultimatum game. Cogn Affect Behav Neurosci. 2012;12(3):419-29.

27. Garfinkel SN, Barrett AB, Minati L, et al. What the heart forgets: Cardiac timing influences memory for words and is modulated by metacognition and interoceptive sensitivity. Psychophysiology. 2013;50(6):505-12.

28. Association AP. Diagnostic and statistical manual of mental disorders. 5th ed. Arlington, VA: American Psychiatric Publishing; 2013.

29. Hoehn-Saric R, McLeod DR, Funderburk F, et al. Somatic symptoms and physiologic responses in generalized anxiety disorder and panic disorder - An ambulatory monitor study. Arch Gen Psychiatry. 2004;61(9):913-21.

30. Gupta MA. Review of somatic symptoms in posttraumatic stress disorder. Int Rev Psychiatry. 2013;25(1):86-99.

31. Hassan I, Ali R. The Association Between Somatic Symptoms, Anxiety Disorders and Substance Use. A Literature Review. Psychiatr Q. 2011;82(4):315-28.

32. Anderson ER, Hope DA. The relationship among social phobia, objective and perceived physiological reactivity, and anxiety sensitivity in an adolescent population. J Anxiety Disord. 2009;23(1):18-26.

33. Greeven A, van Balkom AJLM, van Rood YR, et al. The boundary between hypochondriasis and obsessive-compulsive disorder: A cross-sectional study from the Netherlands. J Clin Psychiatry. 2006;67(11):1682-9.

$34 . \bullet \quad$ Ferrao YA, Shavitt RG, Prado H, et al. Sensory phenomena associated with repetitive behaviors in ob- 
sessive-compulsive disorder: an exploratory study of 1001 patients. Psychiatry Res. 2012;197(3):253-8.

This study characterizes sensory phenomena and links them to different symptom subtypes and comorbidities in a large cohort of OCD patients.

35. Prado HS, Rosario MC, Lee J, et al. Sensory phenomena in obsessive-compulsive disorder and tic disorders: a review of the literature. CNS Spectr. 2008;13(5):425-32.

36. Rosario MC, Prado HS, Borcato S, et al. Validation of the University of Sao Paulo Sensory Phenomena Scale: initial psychometric properties. CNS Spectr. 2009;14(6):315-23.

37. Coles ME, Frost RO, Heimberg RG, et al. "Not just right experiences": perfectionism, obsessive-compulsive features and general psychopathology. Behav Res Ther. 2003;41(6):681-700.

38. Leckman JF, Walker DE, Goodman WK, et al. "Just right" perceptions associated with compulsive behavior in Tourette's syndrome. Am J Psychiatry. 1994;151(5):675-80.

39. Cohen AJ, Leckman JF. Sensory Phenomena Associated with Gilles-De-La-Tourettes Syndrome. J Clin Psychiatry. 1992;53(9):319-23.

40. Leckman JF, Walker DE, Cohen DJ. Premonitory Urges in Tourettes-Syndrome. Am J Psychiatry. 1993;150(1):98-102.

41. Reese HE, Scahill L, Peterson AL, et al. The Premonitory Urge to Tic: Measurement, Characteristics, and Correlates in Older Adolescents and Adults. Behav Ther. 2014;45(2):177-86.

42. O'Sullivan RL, Mansueto CS, Lerner EA, et al. Characterization of trichotillomania - A phenomenological model with clinical relevance to obsessivecompulsive spectrum disorders. Psychiatr Clin N Am. 2000;23(3):587-604.

43. Eckman PS, Shean GD. Habituation of cognitive and physiological arousal and social anxiety. Behav Res Ther. 1997;35(12):1113-21.

44. Mirams L, Poliakoff E, Brown RJ, et al. Interoceptive and exteroceptive attention have opposite effects on subsequent somatosensory perceptual decision making. Q J Exp Psychol (Hove). 2012;65(5):92638.

45. Critchley HD, Wiens S, Rotshtein P, et al. Neural systems supporting interoceptive awareness. Nat Neurosci. 2004;7(2):189-95.

46. Pollatos O, Traut-Mattausch E, Schandry R. Differential effects of anxiety and depression on interoceptive accuracy. Depress Anxiety. 2009;26(2):16773.

47. Van der Does AJW, Antony MM, Ehlers A, et al. Heartbeat perception in panic disorder: a reanalysis. Behav Res Ther. 2000;38(1):47-62.

48. Stevens S, Gerlach AL, Cludius B, et al. Heartbeat perception in social anxiety before and during speech anticipation. Behav Res Ther. 2011;49(2):138-43.
49. Kane MJ. Premonitory urges as "attentional tics" in Tourette's syndrome. J Am Acad Child Adolesc Psychiatry. 1994;33(6):805-8.

50. O'Connor K. A cognitive-behavioral/psychophysiological model of tic disorders. Behav Res Ther. 2002;40(10):1113-42.

51. Garfinkel SN, Critchley HD. Interoception, emotion and brain: new insights link internal physiology to social behaviour. Commentary on: "Anterior insular cortex mediates bodily sensibility and social anxiety" by Terasawa et al. (2012). Soc Cogn Affect Neurosci. 2013;8(3):231-4.

52. Craig AD. How do you feel? Interoception: the sense of the physiological condition of the body. Nat Rev Neurosci. 2002;3(8):655-66.

53. Craig AD. Interoception: the sense of the physiological condition of the body. Curr Opin Neurobiol. 2003;13(4):500-5.

54. Cameron OG. Interoception: the inside story-a model for psychosomatic processes. Psychosom Med. 2001;63(5):697-710.

55. Critchley HD, Harrison NA. Visceral Influences on Brain and Behavior. Neuron. 2013;77(4):624-38.

This review summarizes the neural mechanisms of interoception and the importance of body state signaling for emotion, cognition, and self-representation.

56. Schachter SC, Saper CB. Vagus nerve stimulation. Epilepsia. 1998;39(7):677-86.

57. Cervero F, Connell LA. Distribution of Somatic and Visceral Primary Afferent-Fibers within the Thoracic Spinal-Cord of the Cat. J Comp Neurol. 1984;230(1):88-98.

58. Menetrey D, Basbaum AI. Spinal and Trigeminal Projections to the Nucleus of the Solitary Tract - a Possible Substrate for Somatovisceral and Viscerovisceral Reflex Activation. J Comp Neurol. 1987;255(3):439-50.

59. Price CJ, Hoyda TD, Ferguson AV. The area postrema: A brain monitor and integrator of systemic autonomic state. Neuroscientist. 2008;14(2):182-94.

60. Mertz H. Role of the brain and sensory pathways in gastrointestinal sensory disorders in humans. Gut. 2002;51:I29-33.

61. Sessle BJ, Hu JW, Amano N, et al. Convergence of Cutaneous, Tooth-Pulp, Visceral, Neck and Muscle Afferents onto Nociceptive and Nonnociceptive Neurons in Trigeminal Subnucleus Caudalis (Medullary Dorsal Horn) and Its Implications for Referred Pain. Pain. 1986;27(2):219-35.

62. Isnard J, Guenot $M$, Sindou $M$, et al. Clinical manifestations of insular lobe seizures: a stereo-electroencephalographic study. Epilepsia. 2004;45(9):1079-90.

63. Ostrowsky K, Magnin M, Ryvlin P, et al. Representation of pain and somatic sensation in the human insula: a study of responses to direct electrical cortical stimulation. Cereb Cortex. 2002;12(4):376-85. 
64. Ibanez A, Gleichgerrcht E, Manes F. Clinical effects of insular damage in humans. Brain Struct Funct. 2010;214(5-6):397-410.

65. Nieuwenhuys R. The insular cortex: a review. Prog Brain Res. 2012;195:123-63.

66. Zaki J, Davis JI, Ochsner KN. Overlapping activity in anterior insula during interoception and emotional experience. Neuroimage. 2012;62(1):493-9.

67. Terasawa Y, Fukushima H, Umeda S. How does interoceptive awareness interact with the subjective experience of emotion? An fMRI study. Hum Brain Mapp. 2013;34(3):598-612.

68. Wiebking C, Duncan NW, Tiret B, et al. GABA in the insula - a predictor of the neural response to interoceptive awareness. Neuroimage. 2014;86:10-8.

69. Farb NAS, Segal ZV, Anderson AK. Attentional Modulation of Primary Interoceptive and Exteroceptive Cortices. Cereb Cortex. 2013;23(1):114-26.

70. Simmons WK, Avery JA, Barcalow JC, et al. Keeping the body in mind: Insula functional organization and functional connectivity integrate interoceptive, exteroceptive, and emotional awareness. Hum Brain Mapp. 2013;34(11):2944-58.

71. Chang LJ, Yarkoni T, Khaw MW, et al. Decoding the Role of the Insula in Human Cognition: Functional Parcellation and Large-Scale Reverse Inference. Cereb Cortex. 2013;23(3):739-49.

72. Kurth F, Zilles K, Fox PT, et al. A link between the systems: functional differentiation and integration within the human insula revealed by meta-analysis. Brain Struct Funct. 2010;214(5-6):519-34.

73. Wager TD, and Barrett LF. From affect to control: Functional specialization of the insula in motivation and regulation. Published online at PsychExtra. 2004; http://affective-science.org. Accessed June 13, 2014.

74. Craig AD. How do you feel-now? The anterior insula and human awareness. Nat Rev Neurosci. 2009;10(1):59-70.

75. Menon V, Uddin LQ. Saliency, switching, attention and control: a network model of insula function. Brain Struct Funct. 2010;214(5-6):655-67.

76. Critchley HD. Neural mechanisms of autonomic, affective, and cognitive integration. J Comp Neurol. 2005;493(1):154-66.

77. von Economo C. Eine neue Art Spezialzellen des Lobus cinguli und Lobus insulae. Z Gesamte Neurol Psychiatr. 1926;100:707-12.

78. Butti C, Santos M, Uppal N, et al. Von Economo neurons: clinical and evolutionary perspectives. Cortex. 2013;49(1):312-26.

79. Cauda F, D'Agata F, Sacco K, et al. Functional connectivity of the insula in the resting brain. Neuroimage. 2011;55(1):8-23.

80. Cerliani L, Thomas RM, Jbabdi S, et al. Probabilistic tractography recovers a rostrocaudal trajectory of connectivity variability in the human insular cortex. Hum Brain Mapp. 2012;33(9):2005-34.
81. Phillips ML, Senior C, Fahy T, et al. Disgust-the forgotten emotion of psychiatry. Br J Psychiatry. 1998; 172:373-5.

82. Olatunji BO, Williams NL, Lohr JM, et al. The structure of disgust: domain specificity in relation to contamination ideation and excessive washing. Behav Res Ther. 2005;43(8):1069-86.

83. Starcevic V, Berle D, Brakoulias V, et al. Functions of compulsions in obsessive-compulsive disorder. Aust N Z J Psychiatry. 2011;45(6):449-57.

84. Olatunji BO, McKay D. Disgust and psychiatric illness: have we remembered? Br J Psychiatry. 2007;190:457-9.

85. Harrison NA, Gray MA, Gianaros PJ, et al. The Embodiment of Emotional Feelings in the Brain. J Neurosci. 2010;30(38):12878-84.

86. Wright P, He G, Shapira NA, et al. Disgust and the insula: fMRI responses to pictures of mutilation and contamination. Neuroreport. 2004;15(15):2347-51.

87. Schienle A, Stark R, Walter B, et al. The insula is not specifically involved in disgust processing: an fMRI study. Neuroreport. 2002;13(16):2023-6.

88. Phillips ML, Young AW, Senior C, et al. A specific neural substrate for perceiving facial expressions of disgust. Nature. 1997;389(6650):495-8.

89. Jabbi M, Bastiaansen J, Keysers C. A common anterior insula representation of disgust observation, experience and imagination shows divergent functional connectivity pathways. PLoS One. 2008;3(8):e2939.

90. Wicker B, Keysers C, Plailly J, et al. Both of us disgusted in My insula: the common neural basis of seeing and feeling disgust. Neuron. 2003;40(3):655-64.

91. Calder AJ, Keane J, Manes F, et al. Impaired recognition and experience of disgust following brain injury. Nat Neurosci. 2000;3(11):1077-8.

92. Jackson SR, Parkinson A, Kim SY, et al. On the functional anatomy of the urge-for-action. Cogn Neurosci. 2011;2(3-4):227-43.

This paper proposes conceptual and neuroanatomical models of "urges for action" and discusses their relevance for the understanding of pathologic urges.

93. Bohlhalter S, Goldfine A, Matteson S, et al. Neural correlates of tic generation in Tourette syndrome: an event-related functional MRI study. Brain. 2006;129(Pt 8):2029-37.

94. Nagai M, Kishi K, Kato S. Insular cortex and neuropsychiatric disorders: A review of recent literature. Eur Psychiatry. 2007;22(6):387-94.

95. Etkin A, Wager TD. Functional neuroimaging of anxiety: a meta-analysis of emotional processing in PTSD, social anxiety disorder, and specific phobia. Am J Psychiatry. 2007;164(10):1476-88.

96. Javanmard M, Shlik J, Kennedy SH, et al. Neuroanatomic correlates of CCK-4-induced panic attacks in healthy humans: A comparison of two time points. Biol Psychiatry. 1999;45(7):872-82. 
97. Cameron OG, Huang GC, Nichols T, et al. Reduced gamma-aminobutyric acid(A)-benzodiazepine binding sites in insular cortex of individuals with panic disorder. Arch Gen Psychiatry. 2007;64(7):793-800.

98. Kwon JS, Kim JJ, Lee DW, et al. Neural correlates of clinical symptoms and cognitive dysfunctions in obsessive-compulsive disorder. Psychiatry Res. 2003;122(1):37-47.

99. Cocchi L, Harrison BJ, Pujol J, et al. Functional alterations of large-scale brain networks related to cognitive control in obsessive-compulsive disorder. Hum Brain Mapp. 2012;33(5):1089-106.

100. Stern ER, Fitzgerald KD, Welsh RC, et al. Resting state functional connectivity between fronto-parietal and default mode networks in obsessive-compulsive disorder. PLoS One. 2012;7(5):e36356.

101. Rauch SL, Savage CR, Alpert NM, et al. The functional neuroanatomy of anxiety: a study of three disorders using positron emission tomography and symptom provocation. Biol Psychiatry. 1997;42(6):446-52.

102. Rotge JY, Guehl D, Dilharreguy B, et al. Provocation of obsessive-compulsive symptoms: a quantitative voxel-based meta-analysis of functional neuroimaging studies. J Psychiatry Neurosci. 2008;33(5):405-12.

103. Shapira NA, Liu Y, He AG, et al. Brain activation by disgust-inducing pictures in obsessive-compulsive disorder. Biol Psychiatry. 2003;54(7):751-6.

104. Schienle A, Schafer A, Stark R, et al. Neural responses of OCD patients towards disorder-relevant, generally disgust-inducing and fear-inducing pictures. Int J Psychophysiol : Off J Int Org Psychophysiol. 2005;57(1):69-77.

105. Stein DJ, Arya M, Pietrini P, et al. Neurocircuitry of disgust and anxiety in obsessive-compulsive disorder: a positron emission tomography study. Metab Brain Dis. 2006;21(2-3):267-77.

106. Stern ER, Welsh RC, Fitzgerald KD, et al. Hyperactive error responses and altered connectivity in ventromedial and frontoinsular cortices in obsessive-compulsive disorder. Biol Psychiatry. 2011;69(6):583-91.

107. Marsh R, Horga G, Parashar N, et al. Altered Activation in Fronto-Striatal Circuits During Sequential Processing of Conflict in Unmedicated Adults with Obsessive-Compulsive Disorder. Biol Psychiatry. 2014;75(8):615-22.

108. Stern ER, Welsh RC, Gonzalez R, et al. Subjective uncertainty and limbic hyperactivation in obsessive-compulsive disorder. Hum Brain Mapp. 2013;34(8):1956-70.

109. Huyser C, Veltman DJ, Wolters LH, et al. Functional magnetic resonance imaging during planning before and after cognitive-behavioral therapy in pediatric obsessive-compulsive disorder. J Am Acad Child Adolesc Psychiatry. 2010;49(12):1238-48. 48 e1-5.

110. Roth RM, Saykin AJ, Flashman LA, et al. Event-related functional magnetic resonance imaging of re- sponse inhibition in obsessive-compulsive disorder. Biol Psychiatry. 2007;62(8):901-9.

111. Nakao T, Nakagawa A, Nakatani E, et al. Working memory dysfunction in obsessive-compulsive disorder: A neuropsychological and functional MRI study. J Psychiatr Res. 2009;43(8):784-91.

112. Via E, Cardoner N, Pujol J, et al. Amygdala activation and symptom dimensions in obsessivecompulsive disorder. Br J Psychiatry. 2014;204(1):61-8.

113. Choi JS, Shin YC, Jung WH, et al. Altered brain activity during reward anticipation in pathological gambling and obsessive-compulsive disorder. PLoS One. 2012;7(9):e45938.

114. Jung WH, Kang DH, Han JY, et al. Aberrant ventral striatal responses during incentive processing in unmedicated patients with obsessive-compulsive disorder. Acta Psychiatr Scand. 2011;123(5):376-86.

115. Becker MP, Nitsch AM, Schlosser R et al. Altered emotional and BOLD responses to negative, positive and ambiguous performance feedback in OCD. Soc Cogn Affect Neurosci. 2013;Epub ahead of print.

116. Gu BM, Park JY, Kang DH, et al. Neural correlates of cognitive inflexibility during task-switching in obsessive-compulsive disorder. Brain. 2008;131:155-64.

117. Remijnse PL, Nielen MMA, van Balkom AJLM, et al. Reduced orbitofrontal-striatal activity on a reversal learning task in obsessive-compulsive disorder. Arch Gen Psychiatry. 2006;63(11):1225-36.

118. Woolley J, Heyman I, Brammer M, et al. Brain activation in paediatric obsessive compulsive disorder during tasks of inhibitory control. Br J Psychiatry. 2008;192(1):25-31.

119. Beck JG, Shipherd JC. Repeated exposure to interoceptive cues: Does habituation of fear occur in panic disorder patients? A preliminary report. Behav Res Ther. 1997;35(6):551-7.

120. Lee K, Noda Y, Nakano Y et al. Interoceptive hypersensitivity and interoceptive exposure in patients with panic disorder: specificity and effectiveness. Bmc Psychiatry. 2006;6:32 (see http://www.ncbi.nlm.nih.gov/ pubmed/?term=lee+2006+interoceptive)

121. Kopell BH, Greenberg B, Rezai AR. Deep brain stimulation for psychiatric disorders. J Clin Neurophysiol. 2004;21(1):51-67.

122. Abelson JL, Curtis GC, Sagher O, et al. Deep brain stimulation for refractory obsessive-compulsive disorder. Biol Psychiatry. 2005;57(5):510-6.

123. Greenberg BD, Malone DA, Friehs GM, et al. Threeyear outcomes in deep brain stimulation for highly resistant obsessive-compulsive disorder. Neuropsychopharmacol. 2006;31(11):2384-93.

124. Goodman WK, Foote KD, Greenberg BD, et al. Deep Brain Stimulation for Intractable Obsessive Compulsive Disorder: Pilot Study Using a Blinded, Staggered-Onset Design. Biol Psychiatry. 2010;67(6):535-42. 
125. Denys D, Mantione M, Figee M, et al. Deep brain stimulation of the nucleus accumbens for treatment-refractory obsessive-compulsive disorder. Arch Gen Psychiatry. 2010;67(10):1061-8.

126. Sulzer J, Haller S, Scharnowski F, et al. Real-time fMRI neurofeedback: Progress and challenges. Neuroimage. 2013;76(1):386-99.

127. Caria A, Sitaram R, Veit R, et al. Volitional Control of Anterior Insula Activity Modulates the Response to Aversive Stimuli. A Real-Time Functional Mag- netic Resonance Imaging Study. Biol Psychiatry. 2010;68(5):425-32.

128. Ruiz S, Lee S, Soekadar SR, et al. Acquired selfcontrol of insula cortex modulates emotion recognition and brain network connectivity in schizophrenia. Hum Brain Mapp. 2013;34(1):200-12.

129. Veit $R$, Singh V, Sitaram R, et al. Using real-time fMRI to learn voluntary regulation of the anterior insula in the presence of threat-related stimuli. Soc Cogn Affect Neurosci. 2012;7(6):623-34. 